\title{
Global Inspection, Diagnosis and Repair System for Buildings: Homogenis- ing the Classification of Repair Techniques
}

\author{
Clara Pereira $^{1}$, Jorge de Brito ${ }^{2}$ and José D. Silvestre ${ }^{3}$ \\ ${ }^{1}$ CERIS, Instituto Superior Técnico, Universidade de Lisboa, Av. Rovisco Pais, 1, 1049-001 Lisboa, \\ Portugal, clareira@sapo.pt \\ ${ }^{2}$ CERIS, Instituto Superior Técnico, Universidade de Lisboa, Av. Rovisco Pais, 1, 1049-001 Lisboa, \\ Portugal, jb@civil.ist.utl.pt \\ ${ }^{3}$ CERIS, Instituto Superior Técnico, Universidade de Lisboa, Av. Rovisco Pais, 1, 1049-001 Lisboa, \\ Portugal, jose.silvestre@tecnico.ulisboa.pt
}

\begin{abstract}
Building inspection systems are a useful tool for surveyors, standardising the collection of information. This research is framed within the development of a global inspection system based on twelve expert inspection systems for twelve types of building elements used in the envelope of current buildings. Homogenised classification lists replace the use of several records and unify designations used in fieldwork. A homogenised global classification list of repair techniques comprises, in a single component, all the techniques used to repair defects and eliminate their causes for different types of building elements. It includes curative and preventive repairs, as well as planned maintenance works. Well-defined criteria guided the harmonisation process of a large set of repair techniques in a user-friendly list. For instance, the association of different operations in a single repair technique took into account: similarities between procedures, applicability to one type of building element, solving identical defects and the use of similar repair materials. Considering data from the validation samples of the expert inspection systems, it is observed that techniques " $R$-A1 Cleaning" in painted façades and " $R$-A12 Application of a new (adequate) cladding/finishing coat over the existent/replacement" in wall renders are the most commonly prescribed. The provision of a homogenised classification list of repair techniques is expected to improve the quality of information collected on-site and give a comprehensive view of the most relevant repair techniques used in the envelope of current buildings.
\end{abstract}

Keywords: Building Pathology, Degradation, Inspection Systems, Maintenance, Repair Techniques.

\section{Introduction}

Building inspection systems are useful for surveyors as they (i) help implementing uniform methodologies, (ii) guide the procedures through all the stages, and (iii) use standard designations. In this way, the collection of data on-site and inspection reports become methodic, hence standardising building inspections. This paper addresses the development of a global inspection system for the building envelope by a research team at Instituto Superior Técnico (IST), Universidade de Lisboa (UL), based on individual expert inspection systems (Pereira et al., 2018).

The global inspection system under development at IST-UL is directed at current buildings. It is a broad tool including classification lists, correlation matrices, detailed information on degradation parameters, and an inspection form. It is based on existing expert inspection systems for individual building elements, which implies homogenising the system's components.

The homogenisation of classification lists simplifies the inspection process, replacing several lists with harmonised lists, more manageable to use. In this way, the identification of pathological phenomena in a painted façade or flat roof requires only one list of defects, for instance. 
Additionally, standard designations may improve communication.

In this paper, the methodology of development of the harmonised list of repair techniques in the global inspection system is described, and the proposed classification list is presented. Then, using relative frequency results, the most common repair techniques are analysed. The paper ends summarising the research in concluding remarks.

\section{Classification List of Repair Techniques}

Following the structure of individual inspection systems, the global building inspection system includes a list of repair techniques. It contains techniques used to repair defects and eliminate their causes in the group of building elements taken into account in the inspection system. The particulars of each procedure are described in detail in files of repair techniques, out of the scope of this paper.

\subsection{The Context of Developing a Global Inspection System}

The development of the global building inspection system considered a well-defined range of building elements, corresponding to twelve expert inspection systems previously devised by researchers at IST-UL (Table 1). Those expert inspection systems have an identical structure and were all validated through the inspection of significant samples of building elements, hence providing a solid base for a new global system (Ferraz et al., 2016).

All the selected individual expert inspection systems provide a classification list of repair techniques that may be recommended by surveyors following the detection and diagnosis of defects in visual and detailed bulding inspections. Those lists were based on the literature and experience including curative and Brito, 2010). Curative

ventive repair techniques with a specific defect but instead interventions on
d, act to eliminat planned mair ventive repair techniques may include partial or complete replacement and changes in the charac-

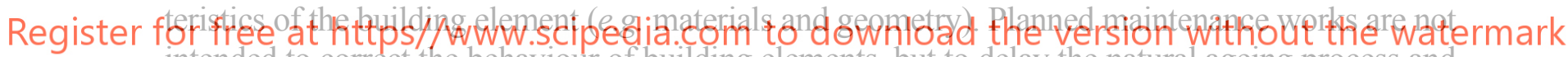
intended to correct the behaviour of building elements, but to delay the natural ageing process and the resulting loss of performance and decrease of the service life.

\subsection{Harmonisation Criteria}

To achieve the goal of having a single list of repair techniques applying to different building elements within the global inspection system, all the expert classification lists had to be collected. Then, going through each list consecutively, the repair techniques referring to the same type of work were merged. For instance, ten out of twelve expert classification lists included cleaning works. Therefore, the global classification list comprises cleaning as one of the repair techniques. After that step, the list of repair techniques was still too long. So, similar techniques were grouped in a single repair technique considering equivalent scopes of intervention, identical procedures, and the resolution of the same kind of phenomena. For instance, different intervention techniques acting on the layers of cladding systems of flat roofs were gathered in a single and broader repair technique referring to the application, repair or replacement of the waterproofing system or separation layer in flat roofs (Conceição et al., 2017). Despite the attempt of decreasing the extent of the global classification list of repair techniques, some specific techniques relating to only a type of building element were kept separately in the list, as no basis for merging applied. 
Table 1. Expert inspection systems developed at IST-UL that were the basis of the global inspection system.

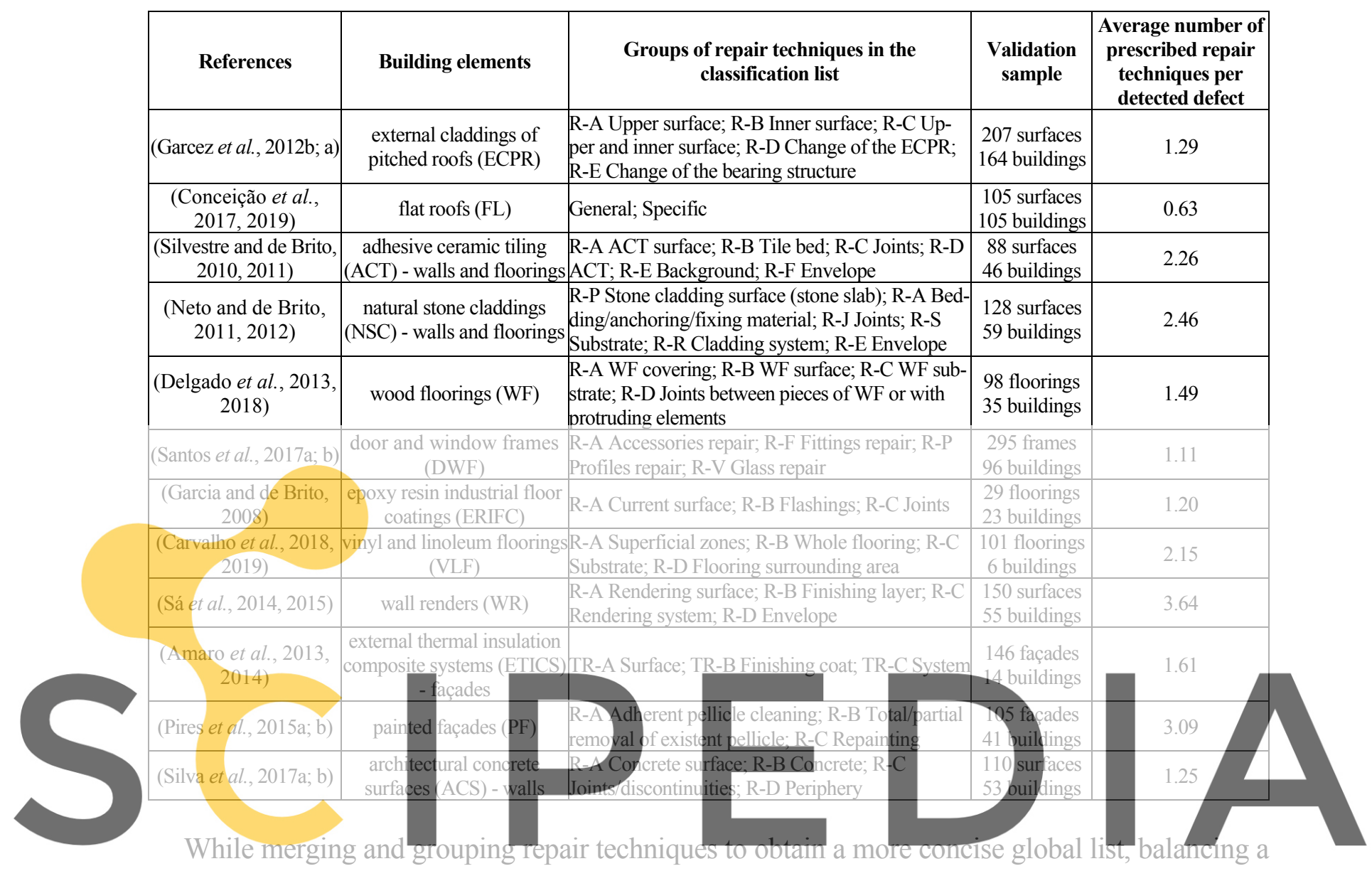

simultaneously elaborate and succinct list is paramount for its broadness and user-friendliness. For

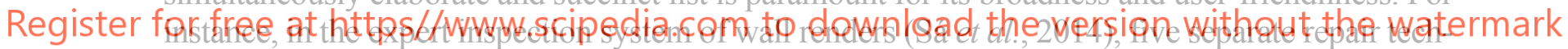

niques were considered to express the idea of applying a new render with higher performance,

namely: application of a reinforced rendering; execution of an external themal insulation composite system (ETICS); execution of a reinforced render coating independent from the bearing wall; application of a render with higher thermal performance; and application of a drainage or corrective rendering. These five types of repair work refer to slightly different operations, but they are all based on the same concept: the existing render is not enough to prevent the occurrence of further defects and should be replaced or overlaid with a new one. Although separately considering each of these techniques may be reasonable in an inspection system only for wall renders, in a global building inspection system, it would hinder the ease of use of the classification list. For this reason, a single repair technique for the application of a higher performance render was proposed.

Still, other repair techniques could not be linked with any others, as they were too specific. For instance, executing a roof slab in pitched roofs is not comparable with other repairs. So, in the global classification list, it was considered separately, although not decreasing the list's complexity.

To guide the harmonisation of the global classification list of repair techniques, taking expert classification lists into account, a set of criteria was determined:

1. Building a brief and concise list of repair techniques without repetitions; 
2. To decrease the extent of the list, coupling techniques in one repair technique considering:

a. The procedure of the repair technique;

b. The applicability of similar repair techniques to a single type of building element;

c. Correcting similar defects;

d. Using repair materials with similar properties;

3. Including all relevant repair techniques in the list, even though some may apply only to a single type of building element, so that the global classification list is comprehensive;

4. Grouping repair techniques into categories according to the intervention area within the cladding systems, namely: surface of the cladding, cladding system, change in the bearing structure/substrate and singularities.

\subsection{Proposed Classification List of Repair Techniques}

Table 2 presents the proposed classification list of repair techniques within a global inspection system. It is organised in four categories, according to the harmonisation criteria. Each technique is identified as constituting a curative (cr) or a preventive repair (pr) or planned maintenance work (pmw). Each category is linked to a code composed of letter R (for repair), a hyphen and a sequential capital letter (from A to D). Each repair technique also corresponds to a code, following that of its category, by adding a sequential number (e.g. R-A1, R-B2, R-C5 and R-D11).

Technique "R-D6 Application/repair/replacement/cleaning of drainage systems/plumbing" results from grouping similar repair techniques. R-D6 is a curative and preventive repair technique used in the drainage and plumbing equipment to eliminate or avoid clogging, accumulation of rainwater and leakages. In the drainage system are menti system refers to the repa (Silvestre and de Brito, 2 the repair of defects in exis
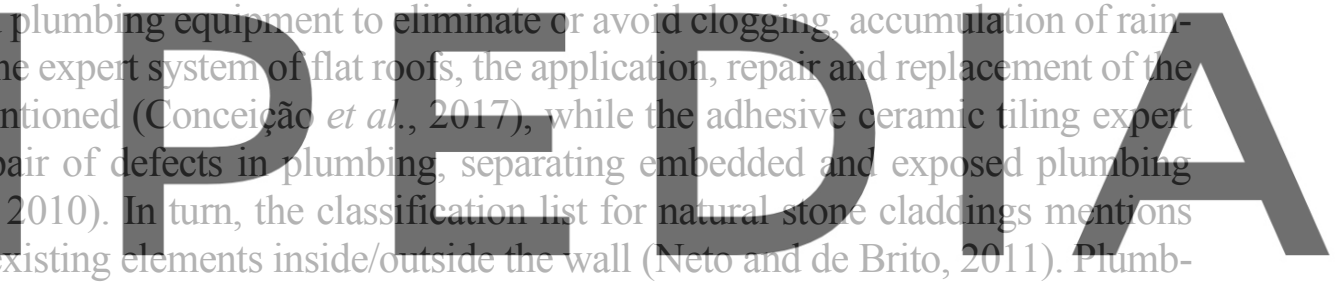

ing is also mentioned for wood floorings (Delgado et al., 2013), while, in door and window frames,

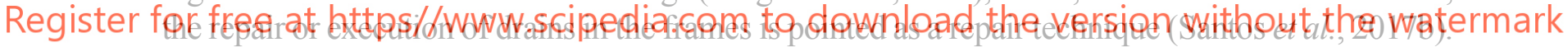

In this context, technique R-D6 is an example of how conciseness influenced the development of the global classification list of repair techniques, as very similar techniques were harmonised in a single and broader repair technique.

Other techniques result from a direct transfer from the expert to the global classification lists. It is the case of "R-D9 Protecting or smoothing of protruding corners or edges", applying to adhesive ceramic tiling, natural stone claddings, wall renders, ETICS and architectural concrete surfaces.

\section{Discussion}

An analysis of the most frequently prescribed repair techniques may be performed using the data from the validation samples of the expert inspection systems (Table 1). To build Figure 1, the absolute frequency of recommendation of each repair technique was considered (Amaro et al., 2014; Carvalho et al., 2019; Conceição et al., 2019; Delgado et al., 2018; Garcez et al., 2012a; Garcia and de Brito, 2008; Neto and de Brito, 2012; Pires et al., 2015b; Sá et al., 2015; Santos et al., 2017a; Silva et al., 2017b; Silvestre and de Brito, 2010), adapting the original repair techniques to the proposed global classification list. Then, those absolute frequencies were divided by the number of detected defects in each validation sample. 
Clara Pereira, Jorge de Brito and José D. Silvestre

Table 2. Proposed classification list of repair techniques within a global inspection system.

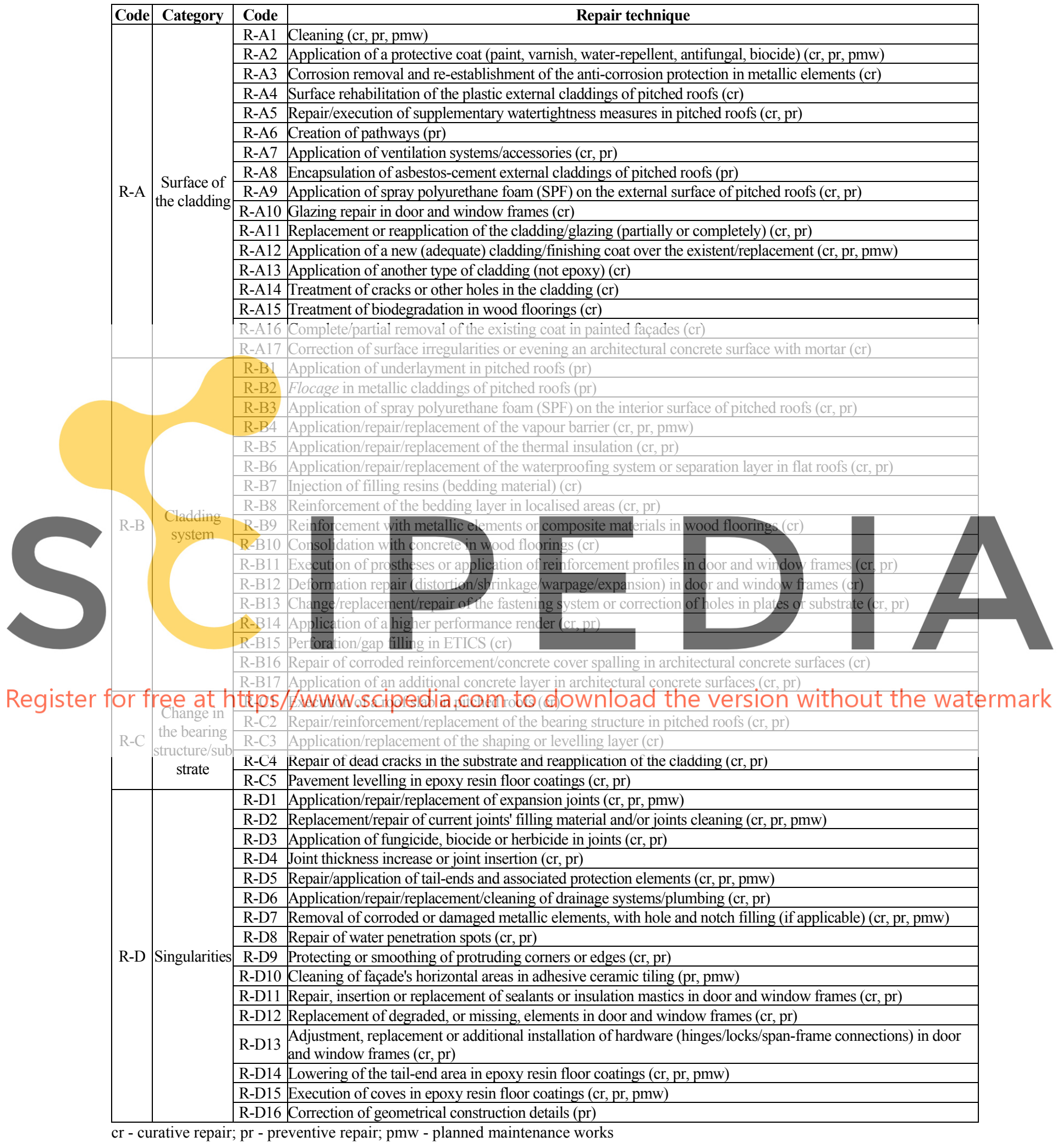



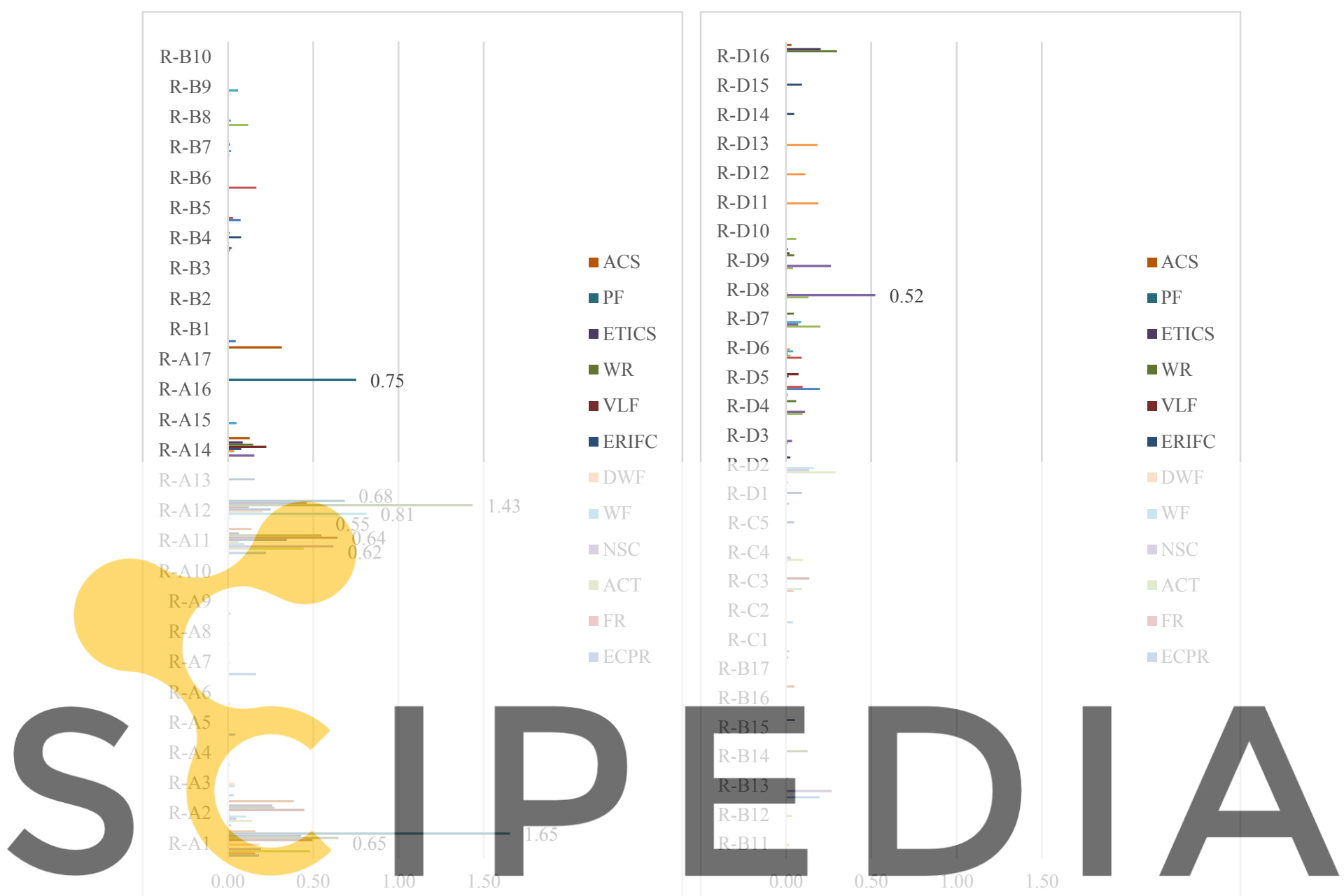

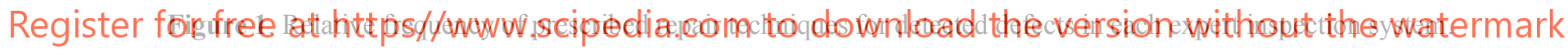

Due to the adaptation to the global classification list, Figure 1 values may result from the sum of absolute frequencies, referring to the union of repair techniques from each expert classification list.

The highest relative frequency in Figure 1 refers to the prescription of "R-A1 Cleaning" in painted façades. R-A1 corresponds to four specific techniques in the expert inspection system of painted façades, which divide the act of cleaning in different operations, namely (Pires et al., 2015a): scrubbing/dusting, manual washing with water and a sponge, washing with a low-pressure water jet, and chemical cleaning. As the result in Figure 1 (1.65) comes from the sum of four absolute frequencies, it exceeds the number of detected defects. It may be interpreted that, in many occurrences, more than one type of cleaning operation was prescribed considering (i) the combination of different activities for better results, or (ii) the prescription of different types of cleaning operations allowing the selection of the best method by decision-makers taking the available equipment and staff into account. Additionally, in the sample of Pires et al. (2015b), the category of anomalies referring to stains represents about $46 \%$ of all detected defects, partially explaining the recommendation of a high number of cleaning operations. Finally, cleaning a painted surface may frequently be considered as preliminary work to prepare the surface for further repairs. 
"R-A12 Application of a new (adequate) cladding/finishing coat over the existent/replacement" is highly recommended in wall renders. Again, the value in Figure 1 (1.43) results from the sum of the absolute frequency of three repair techniques from the wall renders' expert classification list: full/partial replacement of the finishing coat, application of a new finish coat over the existing render, and application of cladding over the existing render (Sá et al., 2014). Considering that inspected renders were protected with a paint coating, and the average age of the sample of buildings is 27 years (Sá et al., 2015), many likely lacked maintenance or repainting according to the standard service life of paint coatings in façades - an average of 8.5 years, according to Chai et al. (2015). Additionally, in the sample of Sá et al. (2015), the cause referring to an irregular repainting periodicity was the most often identified among the group of wear and maintenance faults.

In general, observing Figure 1, it may be concluded that repair techniques in category "R-A Surface of the cladding" are the most commonly prescribed. The outer layer of a building element is the most exposed to aggressive agents. Furthermore, its integrity is of high importance to protect the conditions of the building element and the whole building.

\section{Conclusions}

The use of a single list of repair techniques in the context of building inspections is user-friendly for surveyors and contributes to the standardisation of inspection procedures and the improvement of communication between players in the construction sector. Such a single list of repair techniques may also be useful as it determines the general scope of repair techniques that should be expected

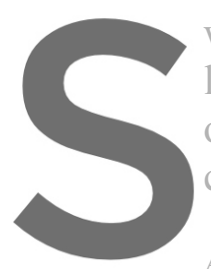
within a maintenance 1 lope. Additionally, analysi owners and maintenan cedures, training work Acknowledgements
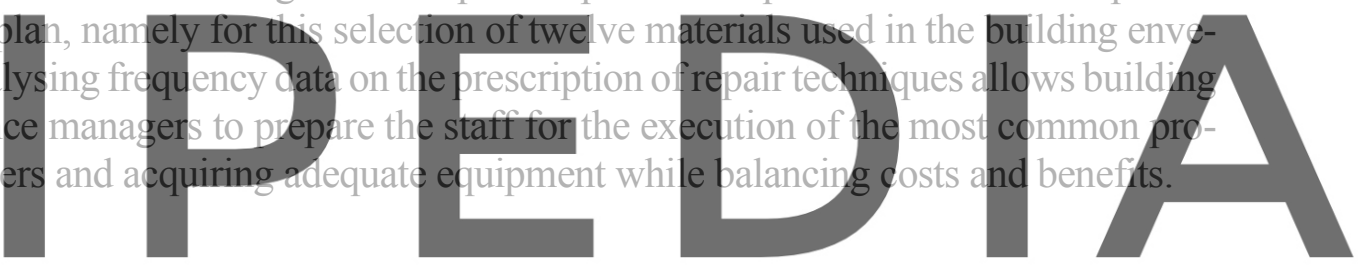

The authors gratefully acknowledge the support of CERIS, from, IST-UL, and the Fundação para a Ciência e a Register for fre era the risks and costs for owners", as well as the FCT PhD Scholarship SFRH/BD/131113/2017.

\section{ORCID}

Clara Pereira: https://orcid.org/0000-0002-9535-1844

Jorge de Brito: https://orcid.org/0000-0001-6766-2736

José D. Silvestre: https://orcid.org/0000-0002-3330-2000

\section{References}

Amaro, B., Saraiva, D., de Brito, J. and Flores-Colen, I. (2013). Inspection and diagnosis system of ETICS on walls. Construction and Building Materials, 47, 1257-1267. doi: 10.1016/j.conbuildmat.2013.06.024

Amaro, B., Saraiva, D., de Brito, J. and Flores-Colen, I. (2014). Statistical survey of the pathology, diagnosis and rehabilitation of ETICS in walls. Journal of Civil Engineering and Management, 20(4), 511-526. doi: $10.3846 / 13923730.2013 .801923$

Carvalho, C., de Brito, J., Flores-Colen, I. and Pereira, C. (2018). Inspection, diagnosis, and rehabilitation system for vinyl and linoleum floorings in health infrastructures. Journal of Performance of Constructed Facilities, 32(6), 04018078. doi: 10.1061/(ASCE)CF.1943-5509.0001229

Carvalho, C., de Brito, J., Flores-Colen, I. and Pereira, C. (2019). Pathology and rehabilitation of vinyl and linoleum floorings in health infrastructures: Statistical survey. Buildings, 9(5), 116. doi: 10.3390/buildings9050116

Chai, C., de Brito, J., Gaspar, P.L. and Silva, A. (2015). Statistical modelling of the service life prediction of painted 
surfaces. International Journal of Strategic Property Management, 19(2), 173-185. doi: 10.3846/1648715X.2015.1031853

Conceição, J., Poça, B., de Brito, J., Flores-Colen, I. and Castelo, A. (2017). Inspection, diagnosis, and rehabilitation system for flat roofs. Journal of Performance of Constructed Facilities, 31(6), 04017100. doi: 10.1061/(ASCE)CF.1943-5509.0001094.

Conceição, J., Poça, B., de Brito, J., Flores-Colen, I. and Castelo, A. (2019). Data analysis of inspection, diagnosis, and rehabilitation of flat roofs. Journal of Performance of Constructed Facilities, 33(1), 04018100. doi: 10.1061/(ASCE)CF.1943-5509.0001252

Delgado, A., de Brito, J. and Silvestre, J.D. (2013). Inspection and diagnosis system for wood flooring. Journal of Performance of Constructed Facilities, 27(5), 564-574. https://doi.org/10.1061/(ASCE)CF.1943-5509.0000342

Delgado, A., Pereira, C., de Brito, J. and Silvestre, J.D. (2018). Defect characterization, diagnosis and repair of wood flooring based on a field survey. Materiales de Construcción, 68(329), 1-13. doi: 10.3989/mc.2018.01817

Ferraz, G.T., de Brito, J., de Freitas, V.P. and Silvestre, J.D. (2016). State-of-the-art review of building inspection systems. Journal of Performance of Constructed Facilities, 30(5), 04016018. doi: 10.1061/(ASCE)CF.1943-5509.0000839

Garcez, N., Lopes, N., de Brito, J. and Sá, G. (2012). Pathology, diagnosis and repair of pitched roofs with ceramic tiles: Statistical characterisation and lessons learned from inspections. Construction and Building Materials, 36, 807-819. doi: 10.1016/j.conbuildmat.2012.06.049

Garcez, N., Lopes, N., de Brito, J. and Silvestre, J. (2012). System of inspection, diagnosis and repair of external claddings of pitched roofs. Construction and Building Materials, 35, 1034-1044. doi: 10.1016/j.conbuildmat.2012.06.047

Garcia, J. and de Brito, J. (2008). Inspection and diagnosis of epoxy resin industrial floor coatings. Journal of Materials in Civil Engineering, 20(2), 128-136. doi: 10.10617(ASCE)0899-1561(2008)20:2(128)

Neto, N. and de Brito, J. (2011). Inspection and defect diagnosis system for natural stone cladding. Journal of Materials in Civil Engineering, 23(10), 1433-1443. doi: 10.1061/(ASCE)MT.1943-5533.0000314.

Neto, N. and de Brito, J. (2012). Validation of an inspection and diagnosis system for anomalies in natural stone cladding (NSC). Construction and Building Materials, 30, 224-236. doi: 10.1016/j.conbuildmat.2011.12.032

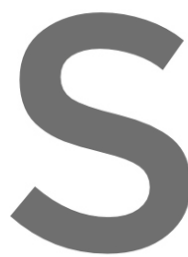

Pereira, C., de Brito, J. and Silvestre, J. the level of detail of the det ogy, Rehabilitation Tec

Pires, R., de Brito, J. and Annart Journal of Performance of

Pires, R., de Brito, J. and Arnar
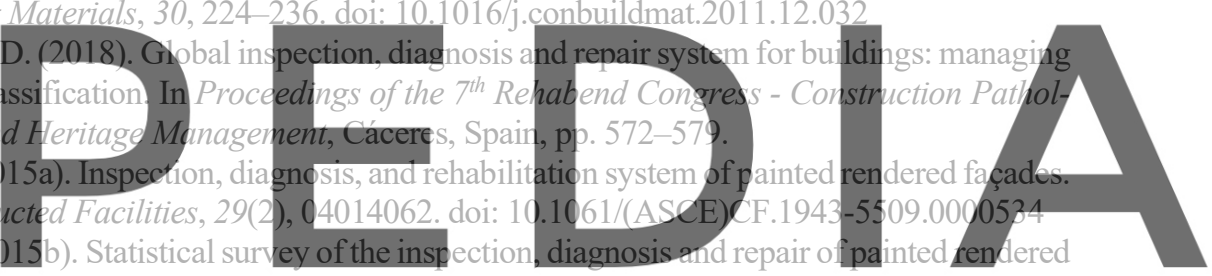
façades. Structure and Infrastructure Engineering, 11(5), 605-618. doi: 10.1080/15732479.2014.890233

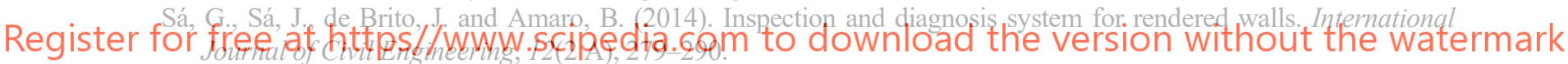

Sá, Gonçalo, Sá, J., de Brito, J. and Amaro, B. (2015). Statistical survey on inspection, diagnosis and repair of wall renderings. Journal of Civil Engineering and Management, 21(5), 623-636. doi: 10.3846/13923730.2014.890666

Santos, A., Vicente, M., de Brito, J., Flores-Colen, I. and Castelo, A. (2017a). Analysis of the inspection, diagnosis, and repair of external door and window frames. Journal of Performance of Constructed Facilities, 31(6), 04017098. doi: 10.1061/(ASCE)CF.1943-5509.0001095

Santos, A., Vicente, M., de Brito, J., Flores-Colen, I. and Castelo, A. (2017b). Inspection, diagnosis, and rehabilitation system of door and window frames. Journal of Performance of Constructed Facilities, 31(3), 04016118. doi: 10.1061/(ASCE)CF.1943-5509.0000992

Silva, C. da, Coelho, F., de Brito, J., Silvestre, J. and Pereira, C. (2017a). Inspection, diagnosis, and repair system for architectural concrete surfaces. Journal of Performance of Constructed Facilities, 31(5), 04017035. doi: 10.1061/(ASCE)CF.1943-5509.0001034

Silva, C. da, Coelho, F., de Brito, J., Silvestre, J. and Pereira, C. (2017b). Statistical survey on inspection, diagnosis and repair of architectural concrete surfaces. Journal of Performance of Constructed Facilities, 31(6), 04017097. doi: 10.1061/(ASCE)CF.1943-5509.0001092

Silvestre, J.D. and de Brito, J. (2011). Ceramic tiling in building façades: Inspection and pathological characterization using an expert system. Construction and Building Materials, 25(4), 1560-1571. doi: 10.1016/j.conbuildmat.2010.09.039

Silvestre, J. Dinis and de Brito, J. (2010). Inspection and repair of ceramic tiling within a building management system. Journal of Materials in Civil Engineering, 22(1), 39-48. doi: 10.1061/(ASCE)0899-1561(2010)22:1(39) 